\title{
DIFFICULT PRESCRIPTIONS.
}

\author{
J. LEON LASCOFF, PHAR. D.
}

I.

B Chloretone $\ldots \ldots \ldots \ldots \ldots \ldots \ldots \ldots \ldots \ldots \ldots$. gr.

Homatropin hydrochloride................ 1 gr.

Aq. Camphoræ....................... 2 dr.

A precipitate was formed which dissolved on warming and later re-precipitated on cooling. We used distilled water, instead of the camphor water, and a clear solution was obtained. After exposing the first mixture in an open vessel for a time, the solution became clear, showing that the precipitation was due to excess of camphor. The liquid being already saturated with the latter cannot dissolve any other salt which is soluble only in this proportion (Chloretone is soluble-1 in 100). This can also be applied to other saturated solutions in which no other salt can be dissolved.

II.

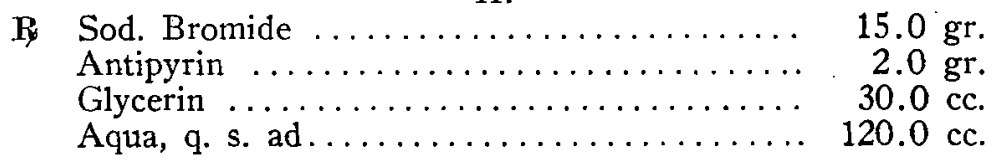

This prescription has very often a green color. This is due to traces of nitrous acid present in glycerin, which reacts with the antipyrin and gives the green color. By the use of Glycerin C. P. a colorless mixture is formed.

III.

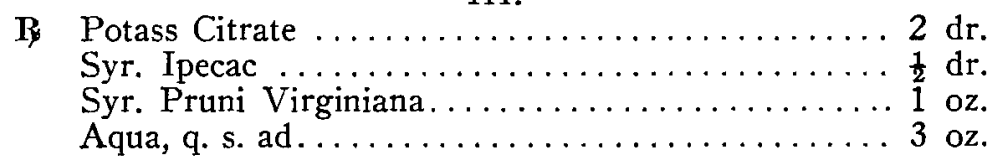

This prescription was sent to us for experiment. We find that the potassium citrate neutralizes the acetic acid, which is added in preparing the syrup of ipecac, to hold in solution the constituents of ipecac, as emetine and others. As the acid is neutralized, the constituents are precipitated. To prevent this, add to the syrup of ipecac, a few drops of acetic acid before mixing. In this case you will get a clear mixture.

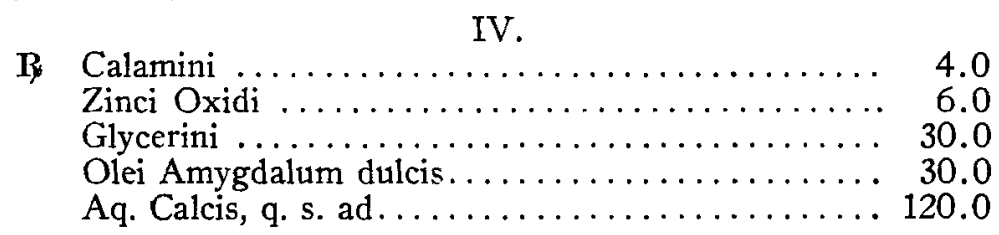

If this prescription is compounded in the order it is written, the oil separates, and no amount of shaking will cause it to mix. To have this prescription properly made, mix the oil with an equal amount of lime water, and shake until an emulsion is formed. Then add a few drops of Fl. Ext. Quillaja and gradually add the remainder of the lime water and the glycerin. Mix the powders in a mortar, and rub up with the mixture. This will form a nice uniform lotion. Even after standing a few days, the powder was still in suspension. 


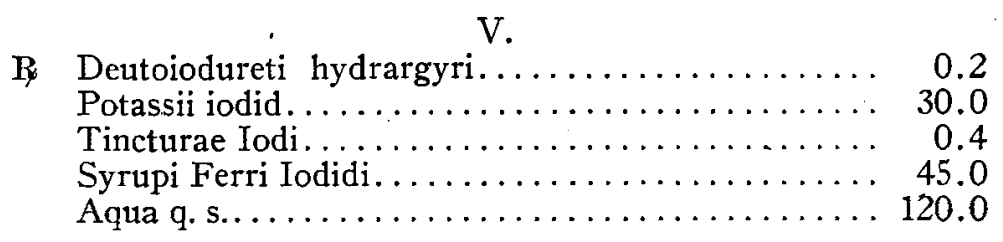

This prescription which is red on first mixing becomes decolorized on standing. The diluted hypophosphorous acid of the syrupus ferri iodidi reacts with the free iodine of the tincture, forming hydriodic acid which is colorless. To have the free iodine which is wanted present in this prescription, therefore the syrup ferrous iodide (1890) which does not contain hyphosphorous acid, should be used. This forms a clear, red mixture, which remains so on standing.

N. B.-The physician informed the patient not to accept the prescription unless it was of red color.

VI.

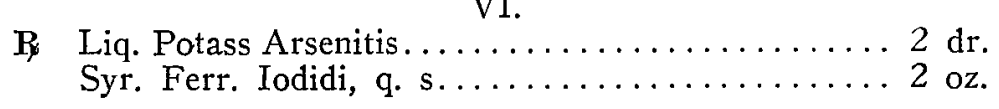

Liq. Potass Arsenitis contains potass. carbonate. The potassium bicarbonate on boiling being converted into carbonate. This,-if the syrup does not contain diluted hypophosphorous acid, as the syrup of U. S. P. 1890,-will react with the ferrous iodide, forming a precipitate of ferrous carbonate, which is converted into ferric hydroxide. To prevent this precipitation, use a syrup containing accurate amount of diluted hypophosphorous acid, or neutralize the Fowler's Solution with diluted hypophosphorous acid before mixing, and you get a perfectly clear mixture.
R Olei Cadini...................... 20.0 cc.
Aqua. Destil, q. s. ad.................. 120.0 cc.

Oleum Cadinii could not be emulsified by the ordinary emulsifying agents, neither could a suspension of the oil in water be obtained. The best way to prepare this is to emulsify the oil with the yolk of an egg, then adding a few drops of F1. Ext. Quillaja (Soap Bark). This helps saponification.

\section{RED GUM.}

\section{JOHN K. THUM.}

Eucalyptus rostrata, sometimes referred to as Australian Kino, and more popnlarly known by the name "Red Gum," is to be found on the market, pharmaceutically, in the form of troches and in a fluid form, misleadingly termed by the manufacturers, a fluidextract.

Like Kino, U. S. P., it contains considerable tannin which makes it extremely valuable as an astringent. The so-called fluidextract has obtained some vogue among throat specialists as a local application in place of the well-known Glyceri- 